\title{
Giro decolonial y colonialidad epistémica en la teoría y los estudios de la organización. Propuesta de investigación en colaboración.
}

\author{
Decolonial turn and epistemic coloniality \\ in the theory and studies of the Organization.
}

Proposal for collaborative research.

\author{
Giovanna M. Mazzotti Pabello \\ MÉJICO
}

\begin{abstract}
Resumen: este trabajo se enmarca en el giro decolonial y los estudios latinoamericanos críticos del management. Apunta al distanciamiento de la colonialidad epistémica de los estudios y la teoría de la organización, así como de la monocultura racional que impone la modernidad/posmodernidad organizacional. Profundiza en la crítica de dos asuntos: las formas en que se ha establecido como científico el conocimiento ordenado y simplificado del mundo (Ibarra-Colado, 2006), y el privilegio epistémico del científico que se sitúa en un imaginario punto cero (Castro-Gómez, 2010) y convierte en universal al conocimiento derivado de la experiencia histórica de 4 o 5 países (Santos, 2010). Se propone la perspectiva decolonial y las prácticas colaborativas de investigaciónacción, para comprender las formas organizativas que no están limitadas por la monocultura racional.
\end{abstract}

Palabras clave: estudios de la organización, colonialidad epistémica, giro decolonial.

Abstract: This work is part of the decolonial turn and critical Latin American studies of management. It points to the distancing of the epistemic coloniality of organization theory and studies and the rational monoculture imposed by organizational modernity/postmodernity. It delves into the critique of two issues: how the ordered and simplified knowledge of the world has been established as the scientific one (Ibarra-Colado, 2006), and the epistemic privilege of the scientist who is situated in an imaginary zero point (Castro-Gómez, 2010) and makes the knowledge derived from the historical experience of 4 or 5 countries universal (Santos, 2010). The paper proposes a decolonial perspective and collaborative action research practices to understand the organizational forms that are not limited by the rational monoculture.

Keywords: studies of the organization, epistemic coloniality, decolonial turn.

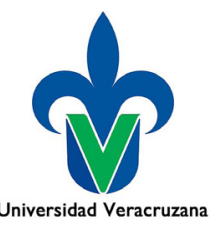

Giovanna M. Mazzotti Pabello es doctora en Estudios Organizacionales por la Universidad Autónoma Metropolitana Unidad Iztapalapa, Méjico. ProfesoraInvestigadora de tiempo completo en el programa de Doctorado en Ciencias Administrativas y Gestión para el Desarrollo en la Facultad de Administración de la Universidad Veracruzana, Campus Xalapa Veracruz, Méjico. 


\title{
Rotação decolonial e colonialidade epistêmica na teoria e nos estudos da organização. \\ Proposta de pesquisa colaborativa.
}

\begin{abstract}
Resumo: este trabalho está enquadrado no giro decolonial e nos estudos latino-americanos críticos da gestão. $\mathrm{O}$ artigo objetiva-se o distanciamento da colonialidade epistêmica dos estudos e da teoria da organização, bem como da monocultura racional que impõe a modernidade/pós-modernidade organizacional. $\mathrm{O}$ artigo se aprofunda na crítica de duas questões: das maneiras pelas quais o conhecimento ordenado e simplificado do mundo foi estabelecido como cientista (Ibarra-Colado, 2006), e o privilégio epistêmico do cientista que está em um ponto imaginário zero (Castro-Gómez, 2010) e torna-se universal ao conhecimento derivado da experiência histórica de 4 ou 5 países (Santos, 2010). A perspectiva decolonial e as práticas colaborativas de pesquisa-ação são propostas para compreender as formas organizacionais que não são limitadas pela monocultura racional.
\end{abstract}

Palavras-chave: estudos da organização, colonialidade epistêmica, giro decolonial.

\section{Introducción}

E

n este trabajo se presentan los avances de lo que inicia como una línea de acción-investigación en el ámbito de los estudios y la teoría de la organización (TO) desde la perspectiva del giro decolonial. Para este fin, se retoman algunos de los criterios desarrollados por distintos investigadores (Mignolo, 2010; Escobar, 2003, 2015; CastroGómez, 2010; Dussel, 2008; Grosfoguel, 2012, 2013; Santos, 2007, 2010; entre otros), quienes desde distintas disciplinas de las ciencias sociales se han planteado formular "alternativas a la modernidad eurocéntrica, tanto en su proyecto de civilización como en sus propuestas epistemológicas" (Pachón, 2008, p. 10), y de este modo "intervenir decisivamente en la discursividad propia de las ciencias modernas para configurar otro espacio para la producción de conocimiento —una forma distinta de pensamiento, «un paradigma otro»- que represente la posibilidad misma de hablar sobre «mundos y conocimientos de otro modo»". (Escobar, 2003, p. 53)

La configuración de otro espacio para la producción de conocimiento, o paradigma otro al que se refiere Escobar (2003), se organiza en torno al programa de investigación Ilamado "modernidad/decolonialidad". Este esfuerzo se conoce también como giro decolonial, puesto que trata de poner en cuestión los criterios a partir de los cuales se establece como referente universal a la modernidad eurocentrada, el modelo de desarrollo expansivo y lineal que trae consigo y la forma de conocimiento basada en un tipo de racionalidad validada en la utilidad de medios y fines que se ha impuesto como legítima. 
En esa dirección, el giro decolonial sitúa la retórica de la modernidad como el anverso de una colonialidad que ella misma produce y la define como un producto histórico-cultural limitado geográficamente en algunos países de Europa central; una modernidad que está basada en una matriz de poder compleja cimentada en la colonización del conocer (epistemología) y del comprender (hermenéutica), sobre la que reposa el control de la economía y de la autoridad. (Quijano, 2000; Mignolo, 2010)

A partir de lo anterior, se han cuestionado los fundamentos teóricos y metodológicos de las disciplinas que constituyen las ciencias sociales y se han transformado "no sólo los contenidos sino los términos mismos de la conversación" (Escobar, 2003, p. 72), de dos modos: uno, al fundamentar la existencia de totalidades históricas y culturales - no circunscritas a la idea de la totalidad moderna - con racionalidades y narrativas propias que se sostienen en formas específicas de conocer y comprender; y dos, al formular una invitación a producir desde los diferentes proyectos políticoepistémicos que existen hoy en el mundo (Dussel, 2008), para llevar a cabo una redefinición de los muchos elementos que han sido apropiados por la modernidad eurocentrada.

En el área de estudios y reflexión sobre las características y la racionalidad específica de las formas de organización de los diversos proyectos político-epistémicos que existen en el mundo, y que no corresponden al esquema impuesto por la modernidad organizacional, poco se ha avanzado en la producción de conocimiento desde el giro decolonial (Zibechi, 2015; Mamani, 2014; Ibarra-Colado, 2006; Esteva, 2012); a pesar de que su incidencia es ampliamente reconocida en el ámbito de distintas disciplinas de las humanidades y las ciencias sociales, tales como la antropología, la sociología, la historia y la filosofía, debido a que ha modificado los términos de la discusión en relación con las cuestiones de raza, género, multi-interculturalidad, pedagogía, lengua-idioma, arteartesanía, cosmovisión-filosofías.

De alguna manera, esto se debe a que los referentes teóricos y metodológicos con los que contamos para abordar, entender y caracterizar a la pluralidad de organizaciones que operan de distintas formas y en diferentes espacios, derivan de que, tal como lo señala Ibarra-Colado (2006) "al abordar los Estudios de la Organización, estamos tratando con una de las formas más importantes de colonialidad epistémica de los últimos 150 años" (p. 466).

En esa dirección, sostenemos que es importante asumir el giro decolonial para analizar los estudios y la teoría de la organización dominante (Ibarra-Colado, 2006) y, de este modo, poner en evidencia la colonialidad 
epistémica del proyecto de modernidad organizacional, que establece como única unidad de análisis a la organización moderna, la que a su vez está ligada a una racionalidad instrumental basada en las cuestiones de productividad, competitividad, calidad y eficiencia (Etzioni, 1975); tal racionalidad se funda en los referentes teóricos analíticos desarrollados por científicos y académicos de 4 o 5 países del mundo -Estados Unidos y tres o cuatro países de Europa- (Santos, 2010) y se han establecido como conocimiento universal gracias a la asunción de un determinado modo de conocer que, para el caso que nos ocupa, se centra en formas de conocimiento ordenado y simplificado del mundo mediante la racionalidad instrumental. (Ibarra-Colado, 2006)

Por ello, en este artículo se toma como punto de partida el giro decolonial y el reconocimiento de la colonialidad epistémica de la teoría y de los estudios de la organización, para comprender las limitaciones que existen al abordar y comprender la diversidad y la pluralidad de las formas de organización que están al margen del proyecto sostenido por la narrativa de la modernidad, y señalar de qué manera dicha modernidad organizacional impuesta ha invisibilizado, marginalizado y colonizado las dinámicas de producción y reproducción de las diversas formas organizativas, cuyas características y lógicas de operaciones no corresponden a la racionalidad productivista e instrumental de la monocultura racional. (Santos, 2007)

Con base en lo anterior, será posible apuntar hacia la necesidad de generar otras formas de conocer y comprender la diversidad organizativa existente, $y$ de reconocer otros tipos de saberes y de conocimientos que se producen y se recrean en las organizaciones en las que el dilema principal, como afirma Esteva (2012), no está en la administración de los recursos o "bienes colectivos" sino en la búsqueda de ampliar los "ámbitos de comunidad", entendidos como formas de vida que los diversos colectivos humanos se organizan para mantener.

Para lograrlo, los fundamentos de la teoría de la organización y del campo de saberes asociados a la modernidad organizacional deben ser profundamente transformados, con la apertura de la jaula epistémica de la modernidad colonial que limita la mirada de lo organizacional a la monocultura racional basada en la lógica productivista, de la escala dominante y del saber instrumental (Santos, 2010). Este esfuerzo exige traspasar las fronteras conceptuales y trascender los límites impuestos por la matriz de poder cimentada en la colonización del conocer (Quijano, 2000) o la colonialidad epistémica. (Ibarra-Colado, 2006) 
De ahí que, a lo largo desde este texto, estaremos insistiendo en construir desde el giro decolonial una toma de distancia de los fundamentos epistémicos de la colonialidad del paradigma moderno que, al responder a la pregunta de qué se puede conocer, oculta el privilegio epistemológico del sujeto cognoscente situado en un no-lugar "por fuera" del mundo, es decir el imaginario punto cero. (Castro-Gómez, 2010)

\title{
La colonialidad epistémica de los estudios y de la teoría de la organización
}

La colonialidad epistémica, de acuerdo con Ibarra-Colado (2006) puede entenderse como los procesos por los cuales se realizó la institucionalización del conocimiento impuesto por las élites angloeuropeas como científico. Según afirma el autor, la teoría de los estudios de la organización se basó primero en la ingeniería, luego en el conocimiento psicológico y finalmente en la gestión. Sus principales efectos han sido la negación, la invisibilización y la marginación de las distintas prácticas y las formas organizativas comunitarias generadas desde otras epistemes, las cuales hay que comprender, como afirma Silvia Rivera Cusicanqui:

\begin{abstract}
como otra atmósfera cognitiva que lleva a reconocer sujetos en el mundo no humano [...] que tienen otra relación de la mano y el cerebro y se manifiesta en el sembrar, el cosechar y el hacer rituales [...] y en el crear comunidad [siendo ésta] también un entorno epistémico de conocimiento en grupo, de procesos de conocer y hacer, de saber hacer con otros... (Ecuador to the World, 2016)
\end{abstract}

Otras epistemes que es preciso comprender sin subsumirlas en las lógicas derivadas de la monocultura racional (Santos, 2010), propias de la teoría y de los estudios de la organización. Ello exige retomar los argumentos críticos que apuntan al tipo de conocimiento generado desde la colonialidad epistémica mencionada (Ibarra-Colado, 2006) y recuperar aquellos que nos permitan reconocer-desconocer los parámetros en los que se sostiene la legitimidad de la disciplina y los "cánones de las ciencias sociales" (Santos, 2010), que se presentan como referentes para determinar la validez de lo que puede ser o no considerado como conocimiento legítimo.

Este punto es central, ya que si hablamos de la necesidad de plantear — desde la perspectiva del giro decolonial_ la posibilidad de generar un conocimiento involucrado con las necesidades y las capacidades de las diversas organizaciones que no pueden ser consideradas como modernas, al no tener el acento puesto en la productividad, la rentabilidad, la planeación o el control, sino en la reproducción de los ámbitos que aseguran la vida colectiva, se debe partir de las preguntas 
que plantea Escobar (2015) y que "son cada vez más acuciantes, aunque la academia «normal» no se dé aún por enterada de su importancia para la construcción del conocimiento: ¿con quién, cómo y desde dónde pensamos? ¿Con qué propósitos? ¿Qué significa pensar con otros [...] en vez de pensar solamente desde los cánones de las ciencias sociales, por críticas que estas parezcan?" (p. 9). Y esto estará o tendrá que estar relacionado con la epistemología, definida en el sentido estricto como "relativa a las cuestiones de cómo sabemos, cómo sabemos que sabemos, en qué justificamos nuestra pretensión de saber y que tan confiable es ese conocimiento". (Gándara, 2011, p. 101)

Esta cuestión no se dirime con el hecho simple de incorporar a la investigación técnicas o herramientas innovadoras diseñadas para generar un conocimiento participativo, o con el hecho de retomar metodologías volcadas a la recuperación de la complejidad existente en los procesos de interacción entre actores-sujetos-objetos (actantes humanos-no humanos), sino que precisa de la reflexión y la reconsideración epistemológica sobre lo que se ha establecido como medio para conocer lo que se ha definido como realidad. De otra forma, se puede pensar que la incorporación del giro decolonial propuesto por el programa modernidad/colonialidad (Escobar, 2003) en la reflexión de las organizaciones implicaría únicamente la ampliación de dicho horizonte hacia el análisis de las organizaciones no modernas, añadiéndolas a la lista de las Ilamadas organizaciones del tercer sector (ONG's, sociedad civil, etc.), cuyo estudio - dicho sea de paso-, en plena sintonía con el discurso reorganizador del sistema mundo neoliberal, no ha hecho sino ampliar el horizonte de la mirada moderna-colonial a espacios organizativos que no habían sido cubiertos por esta.

Por ello, presentamos los argumentos que se sostienen por encima de lo que implícitamente ha sido impuesto como la modernidad organizacional y los medios que se han instituido para hablar acerca de ella. En razón de lo anterior, el punto de partida es la recuperación de las preguntas que plantea Grosfoguel (2013) a partir de la propuesta de Santos (2010), al interrogarse:

¿Cómo es posible que el canon de pensamiento en todas las disciplinas de las ciencias humanas (ciencias sociales y humanidades) en la universidad occidentalizada (Grosfoguel, 2012) se base en el conocimiento producido por unos cuantos hombres de cinco países de Europa occidental (Italia, Francia, Inglaterra, Alemania y los EE.UU.)? ¿Cómo es posible que los hombres de estos cinco países alcanzaran tal privilegio epistémico hasta el punto de que hoy en día se considere su conocimiento superior al del resto del mundo? ¿Cómo lograron monopolizar la autoridad del conocimiento en el mundo? (2013, p. 34) 
Tal monopolio de la autoridad del conocimiento da lugar a la colonialidad epistémica y se fundamenta en la superioridad explicativa por su llamada 'validación científica' (Ibarra-Colado, 2006), que es resultado de la supuesta ausencia de implicaciones subjetivas por parte del sujeto cognoscente; y el hecho de que tal objetividad adquiera el rango de universal se ha producido, según la propuesta del giro decolonial, debido a la instrumentalización del privilegio epistémico del ego-cogito/conquiro (Dussel, 2008) y del imaginario "punto cero" descrito por Castro-Gómez (2010) como ese "no lugar hipotético" desde el cual el científico “ilustrado" mira sin ser mirado y en el que se sitúa el investigador para imponer, desde afuera y por encima, una interpretación sobre los hechos que se afirman a sí mismos como verdaderos.

Este privilegio epistémico, en el caso de los estudios y la teoría de la organización dominante, se convierte —como veremos más adelante-en la colonialidad epistémica a través de la incorporación de la monocultura racional transformada en referente del modelo de organización moderna. El primer aspecto, el sesgo del método 'de validación científica', parte de las cualidades del sujeto cognoscente en tanto ego-cogito/conquiro (Dussel, 2008; Grosfguel, 2013) y, el segundo, de su toma de posición en el imaginario punto cero del que nos habla Castro-Gómez,
[...] según el cual, un observador del mundo social puede colocarse en una plataforma neutra de observación que, a su vez, no puede ser observada desde ningún punto. Nuestro hipotético observador estaría en la capacidad de adoptar una mirada soberana sobre el mundo, cuyo poder radicaría precisamente en que no puede ser observada ni representada. Los habitantes del punto cero (científicos y filósofos ilustrados) están convencidos de que pueden adquirir un punto de vista sobre el cual no es posible adoptar ningún punto de vista. (2010, p. 18)

Por su parte, el ideal del sujeto cognoscente del método, tal como lo señala Dussel (2008), es el 'yo' cartesiano que, al pensar, "puede producir un conocimiento que es «verdadero» más allá del tiempo y el espacio, «universal» en el sentido de que no está condicionado por ninguna particularidad, y «objetivo» entendido como equivalente a «neutralidad»"; lo que, ante la pregunta sobre la validez y coherencia de tales pensamientos con la realidad, encuentra respuesta en "el método del solipsismo [en el que] el sujeto plantea y responde preguntas en un monólogo interno hasta que llega a la certidumbre en el conocimiento". (Grosfoguel, 2013, p. 37)

Así mismo, Dussel (2008) afirma que el ideal del 'yo pienso' de Descartes está política, histórica y geográficamente precedido por 150 años de 'yo conquisto, luego existo', lo que explica el sesgo conquistador del tipo de 
pensamiento que se considera capaz de producir conocimiento universal, sin diálogo y concebido desde un lugar imaginario indeterminado por el tiempo y el espacio, como lo describe Castro-Gómez:

El ideal del científico ilustrado es tomar distancia epistemológica
frente al lenguaje cotidiano - considerado como fuente de error
y confusión-para ubicarse en lo que he denominado el punto
cero. A diferencia de los demás lenguajes humanos, el lenguaje
universal de la ciencia no tiene un lugar específico en el mapa, sino
que es una plataforma neutra de observación a partir de la cual el
mundo puede ser nombrado en su esencialidad. (2010, p.14)

El privilegio epistémico del científico ilustrado tiene otra cara: la inferioridad epistémica; así lo anota Santos (2010) cuando afirma que entre unos y otros hay una línea imaginaria y abismal que divide el universo entre los que están 'de este lado de la línea', que es el mundo de lo humano o la zona del ser, cuyos habitantes tienen derecho a hablar y a existir, y lo que ocurre 'del otro lado de la línea', que es la zona de no-ser, donde las poblaciones son deshumanizadas. La inferioridad epistémica se refleja, como lo afirma Castro-Gómez, en que:

La historia del conocimiento, tal como es representada desde el punto cero, tiene un lugar en el mapa, una geografía específica. Asia, África y América Latina quedan por fuera de esta cartografía [...] Los conocimientos que no se produzcan en esos centros de poder o en los circuitos controlados por ellos, son declarados irrelevantes $y$ «precientíficos». (2010, p.61)

En el caso de la teoría y los estudios de la organización basados en la ingeniería, en el conocimiento psicológico y en la gestión (Ibarra-Colado, 2006), lo antes mencionado delinea el proyecto/concepto de modernidad/ colonialidad, lo que instaura un modelo de organización basado en la monocultura racional que, según Santos (2010, pp. 90-92), se conforma de cinco lógicas, cada una de ellas capaces de producir activamente la inexistencia de la alteridad: la primera deriva de la monocultura del saber y del rigor del saber, está íntimamente ligada a la colonialidad epistémica de la que estamos hablando y se manifiesta en la invalidación de otros conocimientos y saberes.

La segunda se refiere a la monocultura del tiempo lineal, según la cual se afirma que la historia tiene un sentido único —el desarrollo- y se construye en torno a la asunción de que toda organización está -o debería estar - ajustada del mejor modo a la consecución de objetivos racionalmente planteados; con lo cual se niega, por tanto, la existencia de las formas organizativas que perviven y trascienden históricamente a la modernidad. La tercera lógica, que consiste en la naturalización de 
la distribución de poblaciones y la jerarquización de las diferencias, va a manifestarse no solo en la instauración de la primera jerarquización basada en la división del trabajo intelectual y manual, sino también de todas aquellas distribuciones de funciones y tareas prescritas en función del cumplimiento de los objetivos, lo que niega la pertinencia de otras formas de hacer las cosas.

Por su parte, la cuarta lógica corresponde a la monocultura de la escala dominante, que determina la irrelevancia de todas las otras escalas posibles; esta aparece de dos formas principales que son constitutivas de la colonialidad epistémica: la universalidad y la globalidad. La primera resulta de la imposición del imaginario punto cero y la segunda de la imposición de la centralidad de ciertas entidades frente a otras. Por último, se habla de la lógica productivista que se asienta en la monocultura de los criterios de productividad, y está íntimamente ligada a la racionalidad instrumental y a las nociones de eficiencia y eficacia como últimos principios valorativos.

Con base en los argumentos propuestos, consideramos que es posible crear una distancia frente a la colonialidad epistémica de los estudios y la teoría de la organización y asumir - junto con lo que el giro decolonial propone-la necesidad de generar otro tipo de conocimiento, transformador y reflexivo, resultado del diálogo de saberes que amplía el pluriverso e incrementa las posibilidades de abarcar formas organizativas que emanan de entornos cognitivos en los que la comunidad, y no la productividad, es la preocupación principal.

\section{Pasos hacia una epistemología no colonial en los estudios de las organizaciones}

La previa reflexión sobre los aspectos que conforman la colonialidad epistémica en los estudios de la organización nos permite comprender que la diferencia no está ni en el tipo de herramientas, ni en los procedimientos técnicos mediante los cuales se obtiene o se procesa la información, sino en la posición en la que se sitúa el investigador respecto al sujeto colectivo (organización), en los objetivos mismos de la investigación, en los resultados que se buscan, en la finalidad y los procesos que tienen lugar durante el desarrollo del trabajo.

El énfasis está en distinguir entre la postura moderna/colonial de investigación extractiva, de la investigación con giro decolonial. La primera, denominada por Halle (2015) como geopiratería, es en la que el investigador se sitúa "por encima y por fuera" y plantea las preguntas previamente formuladas con base en los intereses marcados por los 
cánones de las ciencias sociales, los que en los estudios y la teoría de la organización están definidos por la monocultura racional; mientras que la investigación con giro decolonial se distancia de la colonialidad epistémica y va a la búsqueda de la construcción de otro tipo de conocimiento basado en la reciprocidad investigativa. Es por ello que es tan importante el reconocimiento de los fundamentos de cómo sabemos, cómo sabemos que sabemos y en qué justificamos nuestra pretensión de saber que, tal como lo menciona Gándara (2011), está más allá de las herramientas o técnicas de investigación que se utilicen.

La construcción de un método de investigación basado en la reciprocidad, y no en el extractivismo de información, es un punto neurálgico para señalar la diferencia radical de las investigaciones cuyo objetivo es propiciar la generación de saberes y de nuevas categorías que funcionen como herramientas útiles para la reflexión y el fortalecimiento del trabajo de los grupos u organizaciones, que se basan en las metodologías de co-labor y de co-teorización con giro decolonial. En ese sentido, Halle (2015) habla de un entendimiento pausado de las complejidades y de un proceso paciente de construir relaciones de confianza, pero, sobre todo, de asumir la responsabilidad de la "reflexión en la acción" que compromete el acompañamiento que el investigador puede hacer, basado en principios políticos convergentes $y$, de este modo, ofrecer algo valioso a la organización para que pueda formarse una asociación de beneficio mutuo. (Halle, 2015, p. 31)

En una investigación colaborativa se pretende hacer un aporte en la producción de subjetividades alternativas y potenciar la capacidad de los sujetos para desarrollar las actividades y los propósitos que persigue. Ello implica desplazar el control de la investigación, del investigador a la intersubjetividad generada en la relación con los actores, a partir de lo cual se esclarece de manera conjunta el objetivo de la investigación. En otras palabras, se trata de transformar el 'qué es importante investigar' según el parámetro del investigador (determinado por las agencias o instituciones que promueven o facilitan la realización de dicha investigación), al 'qué necesita el sujeto colectivo' para potenciar su práctica, reflexionar sobre sus condiciones, generar formas de acción y cumplir con sus propósitos.

En esos términos, se plantea la necesidad de llevar a cabo los trabajos de investigación-acción basados en prácticas de co-labor y de co-teorización en las que participan todos los actores involucrados (el investigador y los miembros de la organización) en un proceso reflexivo de conocimiento, para avanzar hacia la definición de una agenda de investigación coordinada con los actores interesados y así construir de manera conjunta formas de colaboración que den lugar a propuestas alternativas, 
a partir del principio de la reciprocidad investigativa (Montoya, 2015, p. 129). Estas prácticas desafían las tradiciones de la corriente académica dominante (Pearce, 2015) porque implica renunciar a la práctica extractiva de la información 'de la investigación moderna-colonial', y abandonar el método del solipsismo (Grosfoguel, 2013); además permiten considerar seriamente el significado de la creación de un conocimiento acerca de las prácticas y las formas de organización que surgen del pensar con otros, a partir de relaciones de confianza, reciprocidad y horizontalidad y no, como cuestiona Escobar (2015), solamente desde los cánones de las ciencias sociales.

Esta perspectiva permite reconocer que no basta con comprender, a la manera de Gibbons et al. (1997), que el tipo de conocimiento necesario y el modo de "producción" ha cambiado en función de que su forma de validación sea la capacidad para resolver problemas; sino que, como sostiene Escobar (2015, p. 9), las preguntas “¿con quién, cómo y desde dónde pensamos? ¿Con qué propósitos?" son de vital importancia para la construcción de un tipo de conocimiento que permita reconocer la diversidad organizativa que rebasa el modelo impuesto por la modernidad organizacional, sostenido por la colonialidad epistémica de la teoría y los estudios de la organización (Ibarra-Colado, 2006).

Para ello es necesario reconocer que el conocimiento que actualmente tenemos sobre las formas y las prácticas organizativas corresponde a la manera en que la monocultura racional se ha institucionalizado, y a la agenda de investigación que ha impuesto a la gestión como paradigma, todo lo cual debe ser transformado. De ahí la necesidad del cuestionamiento sobre los propósitos reales del quehacer como investigadores y del conocimiento posible que se abre tras la renuncia al privilegio epistémico, a fin de incrementar las posibilidades de las infinitas experiencias del mundo que existen en el presente, organizadas para la sustentabilidad de la vida (Santos, 2015, pp.12- 21).

\section{Conclusiones}

Es necesario continuar con la discusión y la reflexión de lo que hasta aquí se ha planteado en relación con el tipo de conocimiento que se requiere para reconocer la diversidad organizativa que rebasa el modelo impuesto por la modernidad organizacional, sostenido por la colonialidad epistémica de la teoría y los estudios de la organización (Ibarra-Colado, 2006).

No obstante, al analizar de manera crítica el conocimiento con el que actualmente contamos para estudiar a las organizaciones, podemos observar que el proyecto de modernidad que lo contiene está sostenido 
en dos pilares: a) la definición de la organización moderna como unidad de análisis, lo que automáticamente deja fuera del universo a todas aquellas organizaciones otras que teóricamente no cuenten con las características preestablecidas y b) el tipo de conocimiento en el que se sostienen, compuesto por las teorías, los estudios o las críticas sobre el operar de esta unidad de análisis, ha sido desarrollado desde un lugar de enunciación específico - Estados Unidos y tres o cuatro países de Europa-y por científicos que instauran una serie de definiciones sobre la realidad organizacional, desde el privilegio epistémico que les otorga el ego conquiro y el punto cero del método.

A partir de lo expuesto, es posible reconocer que las formas de organización sociales y comunitarias generadoras de otros tipos de conocimiento que existen al margen de los circuitos académicos han sido marginadas y deslegitimadas por la modernidad organizacional, tanto como por la colonialidad epistémica de los estudios y la teoría de la organización, que se ha instituido como conocimiento legítimo con base en los fundamentos de un modo de conocer que instaura el privilegio epistémico.

Por ello, al plantearnos descolonizar el saber y el conocimiento heredado por la modernidad organizacional, se deben poner en cuestión los principios más sagrados de la disciplina y del pensamiento canónico de las academias problematizando la objetividad-neutralidad, que ha sido instaurada por "las estructuras de conocimiento modernas/coloniales como epistemología fundacional de las universidades occidentalizadas" (Grosfoguel, 2013, p. 34); y así plantear el desafío de desarrollar otras formas de conocimiento que permitan coadyuvar en los procesos organizativos y de aprendizaje necesarios para aquellas organizaciones comunitarias y sociales que no corresponden a la lógica con la que se identifican las organizaciones modernas.

En esta dirección, el compromiso que como investigadores tenemos para dejar de repetir la práctica extractiva 'de la investigación modernacolonial'nos exige renunciar al privilegio epistémico del ego conquiro y del punto cero, y pensar en elaborar parámetros de la investigación-acción colaborativa, para hacer un aporte teórico práctico basado en procesos de reflexión, reconfiguración y cambio necesarios para todos los actores involucrados.

Solo de este modo estaremos en condiciones de conocer y reconocer la diversidad organizativa y organizacional que ha sido invisibilizada por la monocultura racional, esta que - como se ha insistido- gira en torno a la unidad de análisis organización-moderna. Se busca sobre todo generar otras pautas de relación y otras formas de conocer que rompan el cerco epistemológico de la modernidad y la colonialidad 
epistémica de los estudios de la organización, y abran la comprensión de un universo organizativo social y comunitario que, a diferencia de la 'perspectiva individualista y acumulativa' — ligada a la sociedad occidental capitalista-, permita ordenar la cooperación de un mayor número de individuos y fomentar relaciones de comunidad para llevar una vida 'con autonomía'. (González, 2011, pp. 83-84)

$$
* * *
$$

\section{Referencias bibliográficas}

1. Castro-Gómez, S. (2010). La hybris del punto cero: ciencia, raza e ilustración en la Nueva Granada (1750-1816). Editorial Pontificia Universidad Javeriana.

2. Dussel, E. (2008). Meditaciones anti-cartesianas: sobre el origen del anti-discurso filosófico de la modernidad. Tabula Rasa, (9), 153-197.

3. Ecuador to the World. (10 de noviembre de 2016). Silvia Rivera sobre la episteme indígena [Video]. Youtube. https://www.youtube.com/watch?v=oOQCTOKfMcg

4. Escobar, A. (2003) Mundos y conocimientos de otro modo. El programa de investigación de modernidad/colonialidad latinoamericano. Tabula Rasa, (1), 51-86.

5. Escobar, A. (2015). Presentación. En Leyva Xochitl et al. Prácticas otras de conocimiento(s). Entre crisis, entre guerras, Tomo I (pp. 9-11). Cooperativa Editorial Retos.

6. Esteva, G. (2012). Regenerar el tejido social de la esperanza. Polis, (33), 1-18.

7. Etzioni, A. (1975) Organizaciones Modernas. Unión Tipográfica Editorial Hispanoamericana.

8. Gándara, M. (2011). El análisis teórico en ciencias sociales: aplicación de una teoría del origen del Estado en Mesoamérica. El Colegio de Michoacán.

9. Gibbons, M., Limoges, C., Nowotny, H., Schwartzman, S., Scott, P. y Trow, M. (1997). La nueva producción del conocimiento. La dinámica de la ciencia y la investigación en las sociedades contemporáneas ( 1 a ed.). Ediciones Pomares - Corredor.

10. González, Í. (2011). Comunidad, sistema de cargos y proyecto social. Una propuesta analítica de sociedades locales en Méjico. AlBR. Revista de Antropología Iberoamericana, 6(1), 81-107.

11. Grosfoguel, R. (2012). El concepto de «racismo» en Michel Foucault y Frantz Fanon: ¿teorizar desde la zona del ser o desde la zona del no-ser? Tabula rasa, (16), 79-102.

12. Grosfoguel, R. (2013). Racismo/sexismo epistémico, universidades occidentalizadas y los cuatro genocidios/epistemicidios del largo siglo XVI. Tabula rasa, (19), 31-58.

13. Halle, Ch. (2015). Entre el mapeo participativo y la'geopiratería': las contradicciones (a veces constructivas) de la antropología comprometida. En Leyva Xochitl, et al. Prácticas otras de conocimiento(s). Entre crisis, entre guerras, Tomo II (pp. 299-320). Cooperativa Editorial Retos. 
14. Ibarra-Colado, E. (2006). Organization Studies and Epistemic Coloniality in Latin America:Thinking Otherness from the Margins. Sage Journals Review, 13(4), 463-488.

15. Mamani, P. (2014). Wiphalas y fusiles. Poder comunal y el levantamiento aymara de Achakachi-Omasuyus (2000-2001). Sol de Paz Pachakuti - FLACSO (Ecuador) Revista Willka.

16. Mignolo, W. (2010). Desobediencia epistémica: retórica de la modernidad; lógica de la colonialidad y gramática de la decolonialidad (1 1 a ed.). Ediciones del Signo.

17. Montoya, R. (2015). Cuando la cultura se convierte en política. En Leyva Xochitl, et al. (Coord). Prácticas otras de conocimiento(s). Entre crisis, entre guerras, Tomo III (pp. 125-141). Cooperativa Editorial Retos.

18. Pachón, D. (2008) Nueva perspectiva filosófica en América Latina: el grupo modernidad/colonialidad. Ciencia Política, 3(5), 8-35.

19. Pearce, J. (2015). Avanzamos porque estamos perdidos. En Leyva Xochitl, et al. (Coord). Prácticas otras de conocimiento(s). Entre crisis, entre guerras, Tomo II (pp. 356-380). Cooperativa Editorial Retos.

20. Quijano, A. (2000). Colonialidad del poder, eurocentrismo y América Latina. En Lander Edgardo (comp.) La colonialidad del saber: eurocentrismo y ciencias sociales. Perspectivas Latinoamericana (pp. 201-246). Consejo Latinoamericano de Ciencias Sociales - CLACSO.

21. Santos, B. (2007) Conocer desde el Sur. Para una cultura política emancipatoria (2a ed.). Consejo Latinoamericana de Ciencias Sociales - CLACSO, CIDES - UMSA, Plural editores.

22. Santos, B. (2010). Para descolonizar Occidente: más allá del pensamiento abismal. Consejo Latinoamericana de Ciencias Sociales - CLACSO, Prometeo Libros.

23. Santos, B. (2015). Prólogo. En Leyva Xochitl, et al. (Coord.) Prácticas otras de conocimiento(s). Entre crisis, entre guerras, Tomo I (pp. 12-22). Cooperativa Editorial Retos.

24. Zibechi, R. (2015). Descolonizar el pensamiento críticoy las rebeldías. Laemancipación como producción de vínculos ( $1^{\mathrm{a}}$ ed.). Ediciones bajo tierra.

\begin{tabular}{|c|l|}
\hline $\begin{array}{c}\text { Para citar } \\
\text { este artículo: }\end{array}$ & $\begin{array}{l}\text { Mazzotti P., G. (2021). Giro decolonial y colonialidad epistémica en la teoría y } \\
\text { los estudios de la organización. Propuesta de investigación en colaboración. } \\
\text { Teuken Bidikay, 12(18), 45-58. doi: 10.33571/teuken.v12n18a2 }\end{array}$ \\
\hline
\end{tabular}

Ge*: AMV. 